\title{
THE ULTRAVIOLET IMAGING TELESCOPE FOR ASTRO 1
}

\author{
T.P. STECHER \\ NASA/Goddard Space Flight Center
}

\begin{abstract}
The Ultraviolet Imaging Telescope (UIT) is a 38-cm Ritchey-Chretien telescope with 2 arc-sec resolution and a 40 arc-min field of view. There are two magnetically-focused image intensifiers which record on film. One has a Cs-I photocathode and 6 filters, the other has a Cs-Te cathode with 5 filters and an objective grating. The film transports contain 1000 frames each of IIa-O film. The point source threshold is $\mathrm{m}(2200 \AA)=22.0$ in a 30 min integration through the widest filter. For extended sources the corresponding mag is 26.0/sq. arc-sec. The UIT complements the instruments on the Hubble Space Telescope in that its field of view is $\sim 200$ times that of the Wide Field Camera, it has extremely good visible light rejection and is more sensitive to diffuse structures in the UV. The UIT is well-suited to locating interesting UV targets for HST follow-up and the study of large regions such as nearby galaxies and clusters.

Astro 1 will point the co-aligned UV instruments at $\sim 200$ targets during its 10 day mission. The UIT science team's primary scientific programs include: The search and survey of the UV sky; SN1987A for the UV light echo; The helium burning and collapsing degenerate stars in star clusters; The structure of highly ionized regions in planetary nebulae and supernova remnants; The physics of massive star formation in spiral structure of galaxies; The interstellar medium of other galaxies; A search for active nuclei in 'normal' galaxies; The very faint regions surrounding galaxies that are produced by interactions, mergers, ejection, or accretion flows; The study of stellar populations of galaxies and galactic evolution, ages and chemical content of galaxies, and the study of large samples of galaxies in nearby clusters for comparison with objects at large lookback times.
\end{abstract}

